system, connected with absence of compensatory growth of activity neutrophils SOD and AFS plasma at increase of oximetabolism in neutrophils, observable at other forms arthrosis. Infrigment of AFS plasma and decrease microbicidal activity neutrophils at ill female with knotes arthrosis complicated sinovitis is supposed to be connected to violation in regulation lymphocytes and granulocytes. At the study of morphological structure of blood ill arthrosis increase of relative quantity lymphocytes and decrease neutrophils was found out at the knotes arthrosis at ill female, that is an attribute of painful process.

The given infrigment of morphological structure of blood at ill female with knotes arthrosis complicated sinovitis correlates with growth redox activity, that is possibly connected to induction aggregation neutrophils under influence of immune complexes. Violation oximetabolism neutrophils at ill female with knotes arthrosis complicated sinovitis is the most expressed in the age of 41-50 years old is probably connected with infrigment of gonad system at the women in period, previous menopause and corresponds to data of the literature on high activity arthrosis in this age. The changes occuring on influence laserotherapy are connected to amplification of activity AFS plasma, that occurs at photochemical reactivate by radiation laser ferum and copper contain ferments, at insignificant influence on oximetabolism neutrophils.

Results The greatest positive dynamics redox regulation of neutrophils and activity antioxidizing ferment systems at ill arthrosis occured at acupuncture treatment, as monotherapy, also in combination with diclofenac. Given changes were combined with normalisation of morphological structure of blood. Thus the periostal acupuncture effect is connected not only with the releasing of pain but also with immunomodulation mechanism normalising the neutrophils activity.

Conclusion

\section{AB0147 DRUG TREATMENT OF OSTEOARTHRITIS: ASPECTS OF SYMPTOM AND STRUCTURE MODIFICATION}

KK Förster, KW Schmid. Department of Clinical Research, Opfermann Arzneimittel GmbH, Wiehl, Germany

\subsection{6/annrheumdis-2001.409}

Background "Osteoarthritis (OA) is a degenerative disease of the joint cartilage. It is of diverse aetiology and obscure pathogenesis. Clinically, OA is characterised by joint pain, tenderness, movement limitation, occasional effusion, and variable degrees of local inflammation, but without systemic effects. Therapeutically, OA is characterised by a lack of specific healing". ${ }^{1}$ Therefore, treatment of $\mathrm{OA}$ is mainly a symptomatic one, a specific treatment, with a demonstrable causal effect on the pathological process, is still on the way to be proven clinically.

Objectives Historically, OA has been viewed as a disease for which little can be done to block its progression. However, the significant increase in understanding the underlying pathologic processes has substantially increased the number of potential targets for drug intervention (Howell et al. 1995). In order to clearly define OA therapies, recently it was agreed to classify OA drugs as follows:

- Symptom modifying drugs (with no detectable effect on the structural changes of the disease).

- Structure modifying drugs (which interfere with the progression of $\mathrm{OA}$ ).
Methods Based on published placebo as well as reference controlled clinical studies, evidence is assessed for the classification of drugs used in the treatment of osteoarthritis.

Results Besides corticosteroids, classical NSAIDs, and analgesics, symptom modifying OA pharmacotherapy includes Ademethionine, ASU, Chondroitin sulfate (CS), Diacerein, Glucosamine sulfate (GS, dona 2oo-S), Hyaluronic acid (HA), and Oxaceprol. All show an anti-inflammatory effect, but unlike classical NSAIDs most of them do not inhibit lip- or cyclo-oxygenase. Therefore, they have been described as nonclassical NSAIDs. ${ }^{2}$

On the other hand, experience based on in vitro studies and animal models shows that some drugs used in the treatment of OA, rather than having a beneficial symptomatic effect, may accelerate or exacerbate the pathological changes of OA (Brandt et al. 1998). This potential drug induced cartilage change for the worse has stimulated interest in pharmacologic agents which may positively influence the pathogenetic mechanisms in OA. In case these agents would be disease modifying (Lequesne et al. 1994) or structure modifying drugs (Dougados et al. 1996). Many substances have already been tested experimentally. However, a specific OA treatment has very rarely been confirmed so far in hypothesis testing clinical studies.

Concerning potential structure modification of CS and HA, there are only a few pilot clinical studies. Concerning GS, it was proven recently that during long-term treatment the drug exerts combined structure-modifying and symptom-modifying effects so that it is to suggest that GS could be a disease modifying agent in osteoarthritis (Reginster et al. 2001).

Conclusion A future challenge will be the design and implementation of further appropriate clinical trials (Dieppe 1994), with longer follow-up and with different designs, to assess whether disease modification leads to reduced need of joint surgery and prolonged time to substantial disability, respectively (Reginster et al. 1996).

\section{REFERENCES}

1 Mankin HJ, Brandt KD, Shulman LE. Workshop on etiopathogenesis of osteoarthritis. Proceedings and recommendations. J Rheumatol. 1986;13:1127-60

2 Förster KK. Drug treatment of osteoarthritis: clinical aspects. In: Grifka J, OgilvieHarris DJ, eds. Osteoarthritis. Berlin Heidelberg New York: Springer-Verlag, 2000: 66-81

3 Reginster JY, Deroisy R, Paul I, Lee RL, Henrotin Y, Giacovelli G, Dacre J, Rovati LC, Gosset C. Long-term effects of glucosamine sulphate on osteoarthritis progression: a randomised, placebo-controlled clinical trial. Lancet 1999;i:251-6

\section{AB0148 CHIROPRACTIC MANAGEMENT OF SHOULDER CAPSULITIS AND KNEE OSTEOARTHRITIS}

AP Rozin. The B.Shine Department of Rheumatology, Rambam Medical Center, Haifa, Israel

10.1136/annrheumdis-2001.410

Background The treatment of osteoarthritis (OA) of the knees and adhesive capsulitis of the shoulders (SAC) is often disappointing. Recent EULAR clinical guidlines emphasise the role of non-pharmacological treatment modalities for knee OA. ${ }^{1}$ Since 1990 some reports confirmed the benefit of chiropractic management for low back pain., ${ }^{2,3}$ The theory of the vertebrone laid by Gutzeit (1951) related vertebrogenic nerve involvement with target tissue pathology. Chiropractic method of Kasyan was devised and originally modified for the treatment of spine, shoulders and degenerative disease of the knees. ${ }^{4,5}$

Objectives To assess the results of modified Kasyan chiropractic treatment for osteoarthritis of the knee and for adhesive capsulitis of the shoulder. 
Methods Twenty nine patients with symptomatic OA of the knees and 37 - with SAC (more than 50\% reduction of passive movements on all directions) were referred to chiropractic clinic following failure of long term conventional therapy (physiotherapy, NSAID's, local steroid injections). On admission very high proportion of each group ( $84 \%$ knees and $86 \%$ shoulder) were found to have "silent spondylosis": L2-4 or C5-T1 tenderness, paravertebral muscle spasm, and positive X-Ray for spondylosis. None had lumbar or cervical pain. Two-three chiropractic sessions per week $(15 \pm 11$ sessions for knee OA and $10 \pm 4-$ for SAC) were applied to both the relevant spine and the affected joint (combined therapy).

Results The results (in percentage of patients) are presented in the Table 1 . Once a month maintenance session may be necessary to establish long term results.

\begin{tabular}{lll} 
Abstract AB0148 Table 1 & $\begin{array}{l}\text { Knee } \\
\text { osteoarthritis } \\
\text { (\% of patients) }\end{array}$ & $\begin{array}{l}\text { Adhesive } \\
\text { capsulitis } \\
\text { (\% of patients) }\end{array}$ \\
\hline Full pain relief & 25 & 36 \\
Full function rehabilitation & 50 & 55 \\
Decrease of 30\% or more in pain & 60 & 80 \\
Initial 30\% or more pain increase & 25 & 13 \\
Increase of pain by the end of the therapy & 10 & 6 \\
Reduction of pain (1-10 Score) & Not estimated & $5.5 \pm 3.5$ \\
Shoulder joint elevation angle enlargement & - & $50 \pm 36$ \\
(degree) & & \\
\hline
\end{tabular}

Conclusion When direct therapy to knee or shoulder fails "silent" spondylosis should be searched. Combined chiropractic therapy is highly effective in both situations.

\section{REFERENCES}

1 Pendleton $A$, Arden $N$, Dougados $M$, et al. EULAR recommendations for the management of knee osteoarthritis (ESCITIS). Ann Rheum Dis. 2000;59:936-44

2 Meade TW, Dyer $S$, Browne W, et al. Randomized comparison of chiropractic and hospital outpatient management for low back pain: results from extended follow up. BMJ 1995;311:349-51

3 Shekelle PG, Coulter I, Hurwitz EL, et al. Congruence between decisions to initiate chiropractic spinal manipulation for low back pain and appropriateness criteria in North America. Ann Intern Med. 1998;129:9-17

4 Kasyan N. Manual therapy for spine osteochondrosis. Moskow Medicine, 1985

5 Rozin A. Chiropractic management of degenerative spine disease. Rheumatic Pain Satellite Symposium, 1999, Freiburg, Germany

\section{AB0149 STUDYING OF THEW EFFICIENCY OF THE SYNVISK IN OSTEOARTROSIS}

L Groppa, M Moshneaga. Research Unit, Chisinau State University of Medicine and Pharmaceutics, Chisinau, Republic of Moldova

\subsection{6/annrheumdis-2001.411}

Background At present for improvement of life quality in patients with osteoartrosis is necessary to solve some problems: precluding of development of degenerative process in cartilage of affected joint, relieving of pain and improvement of the function of the joint. For these aims we studied the efficiency of the chondroprotective drug Synvisk by Boenhringer Ingelheim in 25 patients with arthrosis of the knee in medium age of $61,2 \pm 1,7$ years, 16 women and 8 men, with duration of the disease 5,8 \pm 0,9 years. Conform X-ray data the distribution of the patients by stages of the disease was as follow: I grade ? $20 \%$, II grade ? $64 \%$, and III grade ? $16 \%$. In experimental group the drug was administered intraarticular by blind method in dose of $2,0 \mathrm{ml}$ one time in a week, 3 injections for the course, which were repeated after 6 and 12 months. The control group was matched by gender, duration of disease and X-ray date; in this group was administrated placebo conforming same scheme.

Evaluation of the effectiveness and tolerability of compared drugs was carried out in evolution of clinical (pain, functional index by Lesquesne, volume of movement on affected joints), $\mathrm{X}$ ray parameters and functional methods (ultrasonography, scintigraphy with Te99m.

In the basic group were mentioned decreasing of the pain intensity and improvement of the joint functions after single injection in one third of the patients, while in control groups similar cases were absent. After 3 courses of the treatment in the basic group the effects were obtained in $86,7 \%$ of the patients, including $33,3 \%$ very good effect, in 53,4\% moderate effect and only in $13,3 \%$ the effects were absent. In control group there were no effect in $80 \%$ of the patients, and in $20 \%$ of the patients there were mentioned moderate effects; good effects were are not observed. Thus, a presented result suggests that administration of Synvisk lead to significant improvement of the life quality in the patients with arthrosis of the knee.

Objectives

Methods

Results

Conclusion

\section{AB0150 EFFECTS OF GLUCOSAMINE SULFATE AND NIMESULIDE ON THE PROGRESSSION OF KNEE OSTEOARTHRITIS}

Cl Chirieac, E Rezus, D Stratan, R Chiriac. Rheumatology, Rehabilitation Hospital, lasi, Romania

\subsection{6/annrheumdis-2001.412}

\section{Background}

Objectives The aim of this study was to assess the clinical, radiological and biological efficacy and tolerability of oral glucosamine sulfate associated with a selective COX 2 inhibitor, nimesulide, in patients suffering from Knee osteoarthritis (OA).

Methods There was a 6 month randomised, double blind study which included 35 patients of both sexes, aged between 45 and 72 years, with symptomatic knee OA, with Kellgren and Lawerence radiological scores I-II. Patients were treated only with oral glucosamine sulfate and $100 \mathrm{mg}$ nimesulide daily or with a placebo (PBO) associated with $100 \mathrm{mg}$ nimesulide per day. The main outcome criteria were the degree of spontaneous joint pain and the overall mobility capacity; secondary outcome criteria included the joint space measurement and the levels of biochemical markers of bone and joint metabolism (osteocalcin, deoxypyridoline, nitric oxyde, matrix-metalloproteinases).

Results The results of this study confirm that the association glucosamine sulfate- nimesulide was well tolerated and both reduces significantly pain and increase overall joint mobility; a stabilisation of the femoro-tibial joint narrowing (measured with a digitised image analyzer) was noted in these patients, whereas joint narrowing space increased in placebo-treated patients. in addition, the metabolism of bone and joint assessed by different biochemical parameters also stabilised in patients receiving glucosamine sulfate and nimesulide combination, while remained unchanged in the PBO patients. 